\title{
A note on towers of function fields over finite fields
}

\section{Ferruh Özbudak \& Michael Thomas}

To cite this article: Ferruh Özbudak \& Michael Thomas (1998) A note on towers of function fields over finite fields, Communications in Algebra, 26:11, 3737-3741, DOI: $10.1080 / 00927879808826370$

To link to this article: http://dx.doi.org/10.1080/00927879808826370

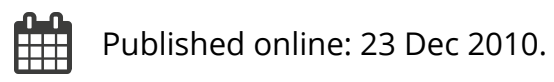

Submit your article to this journal $\pi$

山 Article views: 27

Q View related articles $₫$ 


\title{
A NOTE ON TOWERS OF FUNCTION FIELDS OVER FINITE FIELDS
}

\author{
FERRUH ÖZBUDAK AND MICHAEL THOMAS
}

Bilkent University, Department of Mathematics, 06533 Ankara, Turkey

E-mail address: ozbudakeren.bilkent.edu.tr

UNiversität GH Essen, FB 6 Mathematik und Informatik, D-45117 Essen, Germany

E-mail address: michael.thomas@uni-essen.de

\begin{abstract}
For a tower $F_{1} \subseteq F_{2} \subseteq \cdots$ of algebraic function fields $F_{i} / F_{q}$, define $\lambda:=\lim _{i \rightarrow \infty} N\left(F_{i}\right) / g\left(F_{i}\right)$, where $N\left(F_{i}\right)$ is the number of rational places and $g\left(F_{i}\right)$ is the genus of $F_{i} / F_{q}$. The purpose of this note is to calculate $\lambda$ for a class of towers which was studied in [1], [2] and [3].
\end{abstract}

\section{INTRODUCTION}

Let $\mathbb{F}_{q}$ be a finite field with $q$ elements and $F / \mathbb{F}_{q}$ an algebraic function field, i.e. an algebraic extension of the rational function field $\mathbb{F}_{q}(x)$ of finite degree such that $\mathbb{F}_{q}$ is algebraically closed in $F$. We denote by $N(F)$ the number of rational places of $F / \mathbb{F}_{q}$ and by $g(F)$ the genus of the function field. Weil's theorem states that

$$
|N(F)-(q+1)| \leq 2 g(F) q^{1 / 2}
$$

Fixing $q$, for large genera $g$ this bound could be improved. Namely let $N_{q}(g)=\max \left\{N(F) \mid F\right.$ is a function field over $\mathbb{F}_{q}$ of genus $\left.g\right\}$ and $A(q)=$

This paper was written while the first author was visiting the University of Essen under a grant of TÜBITTAK (Turkish Scientific and Technological Research Council).

Correspondence to the first author. 
$\lim \sup _{g \rightarrow \infty} N_{q}(g) / g$, then by Drinfeld-Vladut bound

$$
A(q) \leq \sqrt{q}-1 .
$$

If $q$ is a square, Ihara and Tsfasman-Vladut-Zink proved that

$$
A(q)=\sqrt{q}-1
$$

If $q$ is not square, the exact value of $A(q)$ is unknown. Serre showed

$$
A(q) \geq c \log q>0 \text { for all } q
$$

with some small constant $c>0$.

A tower of function fields over $\mathbb{F}_{q}$ is a sequence $\mathcal{F}=\left(F_{1}, F_{2}, \ldots\right)$ of function fields $F_{i} / \mathbb{F}_{q}$ having the following properties: (i) $F_{1} \subseteq F_{2} \subseteq F_{3} \subseteq \cdots$, (ii) for every $n \geq 1$, the extension $F_{n+1} / F_{n}$ is separable of degree $>1$, and (iii) $g\left(F_{j}\right)>1$ for some $j \geq 1$. Let $\lambda(\mathcal{F}):=\lim _{n \rightarrow \infty} N\left(F_{n}\right) / g\left(F_{n}\right) . \mathcal{F}$ is called asymptotically good if $\lambda(\mathcal{F})>0$.

It is clear that $\lambda(\mathcal{F}) \leq A(q)$. Garcia-Stichtenoth-Thomas [2] have recently given examples for any $q=p^{e}, e \geq 2$ such that $\lambda(\mathcal{F}) \geq \frac{2}{q-2}$. Namely they constructed a tower of function fields over $\mathbb{F}_{q}, q=p^{e}$, where $F_{n}=\mathbb{F}_{q}\left(x_{1}, \ldots, x_{n}\right)$ and

$$
x_{i+1}^{m}+\left(x_{i}+1\right)^{m}=1, i=1, \ldots, n-1, m=\frac{p^{e}-1}{p-1} .
$$

It would be interesting if the actual value of $\lambda(\mathcal{F})$ was large.

Thomas [3] showed $\lambda(\mathcal{F})=\frac{2}{q-2}$ for a few fixed values of $q$.

In this note we prove the equality for a class of towers for any value of $q$ when $q$ is a square.

Theorem 1.1. Let $\mathbb{F}_{q^{2}}$ be a finite field with $q^{2}$ elements. Let $F_{n}=$ $\mathbb{F}_{q^{2}}\left(x_{1}, x_{2}, \ldots, x_{n}\right)$ be the algebraic function field where

$$
x_{i+1}^{q+1}+\left(x_{i}+1\right)^{q+1}=1, i=1,2, \ldots, n-1 .
$$

Let $\mathcal{F}$ be the tower of function fields over $\mathbb{F}_{q^{2}}$ given by $\mathcal{F}=\left(F_{1}, F_{2}, \ldots, F_{n}, \ldots\right)$. Then

$$
\lambda(\mathcal{F})=\frac{2}{q^{2}-2} .
$$

2. Proof of THE THEOREM

Let $\mathbb{P}_{F_{n}}$ denote the set of places of $F_{n}, n \geq 1, P_{\infty}$ be the place of $F_{1}$ where $v_{P_{\infty}}\left(x_{1}\right)=-1$. Let

$$
S(\mathcal{F})=\left\{P \in \mathbb{P}_{F_{1}} \mid P \text { is ramified in } F_{n} / F_{1} \text { for some } n \geq 2\right\} .
$$

It is known that ([2], Example 2.3) 


$$
S(\mathcal{F}) \subseteq\left\{P \in \mathbb{P}_{F_{1}} \mid P \text { is a rational place and } P \neq P_{\infty}\right\}
$$

Let

$$
A_{n}=\sum_{\substack{P \in \mathbb{P}_{F_{1}} \\ P \neq P_{\infty}}} \sum_{\substack{P^{\prime} \\ P^{\prime} \mid \mathbb{P}_{F_{n}}}} P^{\prime} .
$$

Claim. $\lim _{n \rightarrow \infty} \frac{\operatorname{deg} A_{n}}{(q+1)^{n}}=0$.

The claim shows the equality of two sets in 2.2 , since otherwise there would be a finite place which is unramified in all extensions and hence the limit would be positive.

By Riemann-Hurwitz genus formula

$$
2 g\left(F_{n}\right)-2=\left[F_{n}: F_{1}\right]\left(2 g\left(F_{1}\right)-2\right)+\operatorname{deg} \operatorname{Diff}\left(F_{n} / F_{1}\right) .
$$

From the claim above, more precisely from the equality of the two sets in 2.2 we have

$$
\operatorname{deg} \operatorname{Diff}\left(F_{n} / F_{1}\right)=\left[F_{n}: F_{1}\right] q^{2}-\operatorname{deg} A_{n}
$$

and therefore

$$
g\left(F_{n}\right)=\left[F_{n}: F_{1}\right]\left(g\left(F_{1}\right)-1\right)+\frac{q^{2}\left[F_{n}: F_{1}\right]}{2}-\frac{\operatorname{deg} A_{n}}{2}+1 .
$$

Moreover since $P_{\infty}$ splits completely in all extensions $F_{n} / F_{1}$ we have $\left[F_{n}: F_{1}\right] \leq$ $N\left(F_{n}\right) \leq\left[F_{n}: F_{1}\right]+\operatorname{deg} A_{n}$. Consequently our claim also proves the theorem since $\left[F_{n}: F_{1}\right]=(q+1)^{n-1}$.

Now we prove the claim. For $\alpha, \beta \in \mathbb{F}_{q}$ let $f(\alpha, \beta)=\#\left\{x \in \mathbb{F}_{q^{2}} \mid x^{q+1}=\alpha, x^{q+1}+\right.$ $\left.x^{q}+x=-\beta\right\}$. Then

$\#\left\{\left(x_{1}, x_{2}\right) \in \mathbb{F}_{q^{2}} \times \mathbb{F}_{q^{2}} \mid x_{2}^{q+1}=1-\left(x_{1}+1\right)^{q+1}\right\}=\sum_{\alpha_{1} \in \mathbb{F}_{q}} \sum_{\beta_{2} \in \mathbb{F}_{q}} \sum_{\beta_{2} \in \mathbb{F}_{q}} f\left(\alpha_{1}, \beta_{1}\right) f\left(\beta_{1}, \beta_{2}\right)$ since $x_{2}^{q+1}=-\left(x_{1}^{q+1}+x_{1}^{q}+x_{1}\right)$. Similarly

$$
\begin{gathered}
\#\left\{\left(x_{1}, x_{2}, x_{3}\right) \in \mathbb{F}_{q^{2}} \times \mathbb{F}_{q^{2}} \times \mathbb{F}_{q^{2}} \mid x_{2}^{q+1}=1-\left(x_{1}+1\right)^{q+1} \text { and } x_{3}^{q+1}=1-\left(x_{2}+1\right)^{q+1}\right\} \\
=\sum_{\alpha_{1} \in \mathbb{F}_{q}} \sum_{\beta_{1} \in \mathbb{F}_{q}} \sum_{\beta_{2} \in \mathbb{F}_{q}} \sum_{\beta_{3} \in \mathbb{F}_{q}} f\left(\alpha_{1}, \beta_{1}\right) f\left(\beta_{1}, \beta_{2}\right) f\left(\beta_{2}, \beta_{3}\right)
\end{gathered}
$$

By induction

$$
\operatorname{deg} A_{n}=\sum_{\alpha \in F_{q}} \sum_{\beta \in F_{q}} f^{n}(\alpha, \beta)
$$

where $f^{i+1}(\alpha, \beta)=\sum_{h \in F_{q}} f^{i}(\alpha, h) f(h, \beta) i \geq 1$.

Let $h:\{1,2, \ldots, q\} \rightarrow \mathbb{F}_{q}$ be a bijection such that $h(1)=1$ and $h(q)=0$. Define $G:=\left[G_{i, j}\right]_{1 \leq i \leq q, 1 \leq j \leq q}$ where $G_{i, j}=f(h(i), h(j))$. Considering $G: \mathbb{C}^{q} \rightarrow \mathbb{C}^{q}$ and using $L_{1}$ norm we have $\|G\|=\max _{1 \leq j \leq n} \sum_{i=1}^{q}\left|G_{i, j}\right|$ (see for example [4] page 165). 
We show $\left\|G^{3}\right\|<(q+1)^{3}$ which finishes the proof since $\operatorname{deg} A_{n}=\sum_{i=1}^{q} \sum_{j=1}^{q} G_{i, j}^{n}$. Firstly observe that $0 \leq G_{i, j} \leq 2$. The right hand side follows from the fact that if $a, b \in \mathbb{F}_{q}$ and $f(x)=\operatorname{gcd}\left(x^{q+1}+a, x^{q+1}+x^{q}+x+b\right)$, then $\operatorname{deg} f \leq 2$. Moreover

$$
\sum_{i=1}^{q} G_{i, j}=\left\{\begin{array}{cl}
q+1 & \text { if } j \neq 1 \\
1 & \text { if } j=1
\end{array}\right.
$$

since

$$
\begin{aligned}
\sum_{i=1}^{q} G_{i, j} & =\#\left\{x \in \mathbb{F}_{q^{2}} \mid x^{q+1}+x^{q}+x=-h(j)\right\} \\
& =\#\left\{x \in \mathbb{F}_{q^{2}} \mid(x+1)^{q+1}=1-h(j)\right\} \\
& =\#\left\{x \in \mathbb{F}_{q^{2}} \mid x^{q+1}=1-h(j)\right\} .
\end{aligned}
$$

In fact $G_{i, 1}=\left\{\begin{array}{ll}1 & \text { if } i=1, \\ 0 & \text { if } i \neq 1 .\end{array}\right.$ Similarly

(2.4) $\sum_{j=1}^{q} G_{i, j}=\left\{\begin{array}{cl}q+1 & \text { if } i \neq q, \\ 1 & \text { if } i=q,\end{array} \quad\right.$ and $\quad G_{q, j}= \begin{cases}1 & \text { if } j=q \\ 0 & \text { if } j \neq q\end{cases}$

Using 2.3 we get

$$
\begin{aligned}
\sum_{i=1}^{q} G_{i, j}^{2} & =\sum_{i=1}^{q} \sum_{l=1}^{q} G_{i, l} G_{l, j}=\sum_{l=1}^{q} G_{l, j} \sum_{i=1}^{q} G_{i, l} \\
& =(q-1) \sum_{l=1}^{q} G_{l, j}-q G_{1, j} \\
& =\left\{\begin{array}{cc}
(q+1)^{2}-q G_{1, j} & \text { if } j \neq 1, \\
1 & \text { if } j=1 .
\end{array}\right.
\end{aligned}
$$

Moreover we also get

$$
\begin{aligned}
\sum_{i=1}^{q} G_{i, j}^{3} & =\sum_{i=1}^{q} \sum_{l=1}^{q} G_{i, l} G_{l, j}^{2}=\sum_{l=1}^{q} G_{l, j}^{2} \sum_{i=1}^{q} G_{i, l} \\
& =(q+1) \sum_{l=1}^{q} G_{l, j}^{2}-q G_{1, j}^{2} \\
& =\left\{\begin{array}{cc}
(q+1)^{3}-q(q+1) G_{1, j}-q G_{1, j}^{2} & \text { if } j \neq 1 \\
1 & \text { if } j=1 .
\end{array}\right.
\end{aligned}
$$

However there exists no $2 \leq j \leq q$ such that $G_{1, j}^{2}=0$. Indeed if $G_{1, j}^{2}=0$, then

$$
G_{1, l} G_{l, j}=0 \text { for } l=1, \ldots, q
$$

since the entries are nonnegative. Moreover the entries are bounded from above by 2 and using the properties 2.3 and 2.4 , we get $G_{1, l}=0$ for at most $\frac{q-1}{2}$ many values of $l$ and $G_{l, j}=0$ for at most $\frac{q-1}{2}$ many values of $l$. This gives a contradiction to $G_{1, j}^{2}=0$ and completes the proof.

\section{ACKNOWLEDGMENTS}

We would like to thank Arnolda Garcia, Henning Stichtenoth, and Fernando Torres for the stimulating conversations. Moreover the first author thanks to Fachbereich 6 Universität Essen for their hospitality.

\section{REFERENCES}

[1] Garcia A. and Stichtenoth H., "Asymptotically good towers of function fields over finite felds", C.R. Acad. Sci. Paris 322, Ser. I, pp. 1067-1070, 1996. 
[2] Garcia A., Stichtenoth H. and Thomas M., "On towers and composita of towers of function fields over finite fields", Finite Fields and Their Applications, vol. 3, no. 3, pp. 257-274, 1997.

[3] Thomas M, "Türme und Pyramiden algebraischer Funktionenkörper", Ph.D. Dissertation, University of Essen, 1997.

[4] Elaydi S. N., "An introduction to difference equations", Springer-Verlag, New York, 1996.

Received: June 1997

Revised: February 1998 